\title{
Design of a Mu-Near-Zero metamaterial for reducing the backward magnetic flux leakage of a wireless power transfer system
}

\author{
In-Gon Lee ${ }^{1}$, Nam Kim ${ }^{2}$, In-Kui Cho ${ }^{3}$, and Ic-Pyo Hong ${ }^{1 \text { a) }}$ \\ ${ }^{1}$ Department of Information \& Communication Eng., Kongju National University, \\ 1223-4 Cheonan-dae-ro, Cheonan 31080, Republic of Korea \\ 2 Department of Electronics Engineering, Chungbuk National University, \\ Cheongju, 28644, Republic of Korea \\ ${ }^{3}$ Electronics and Telecommunications Research Institute,
} Daejeon, 34129, Republic of Korea

a)iphong@kongju.ac.kr

\begin{abstract}
This study proposed a design for a metamaterial that has the Mu-Near-Zero property in a certain frequency band in order to reduce the magnetic flux leakage of a wireless power transfer system. The proposed structure had spiral coils arranged in a strip line on a dielectric substrate and used a capacitor connected in parallel to induce magnetic resonance and attain the Mu-Near-Zero property. In this way, magnetic fields are reflected and thus become less likely to leak to the rear of the coils. To verify the performance of the proposed structure, the designed metamaterial was positioned at the rear of the transmission coil, and the efficiency of the transmission and receiver coils and the strength of the leaking magnetic fields were observed. The transmission efficiency at a $0.3 \mathrm{~m}$ distance between the transmission and receiver coils was $40.8 \%$, which was similar to that of the conventional structures, and the reduction of magnetic flux leakage improved by a maximum of $79.7 \%$.
\end{abstract}

Keywords: wireless power transfer, magnetic flux leakage, metamaterial Classification: Microwave and millimeter-wave devices, circuits, and modules

\section{References}

[1] S. Y. R. Hui, et al.: "A critical review of recent progress in mid-range wireless power transfer," IEEE Trans. Power Electron. 29 (2014) 4500 (DOI: 10.1109/ TPEL.2013.2249670).

[2] X. L. Chen, et al.: "Human exposure to close-range resonant wireless power transfer systems as a function of design parameters," IEEE Trans. Electromagn. Compat. 56 (2014) 1027 (DOI: 10.1109/TEMC.2014.2308013).

[3] R. Bosshard, et al:: "Optimized magnetic design for inductive power transfer coils,” APEC (2013) 1812 (DOI: 10.1109/APEC.2013.6520541). 
[4] F. Wen and X. Huang: "Optimal magnetic field shielding method by metallic sheets in wireless power transfer system," Energies 9 (2016) 733 (DOI: 10. 3390/en9090733).

[5] D. Mishra, et al.: "Nanomagnetic structures for inductive coupling and shielding in wireless charging applications," ECTC (2015) 941 (DOI: 10.1109/ ECTC.2015.7159707).

[6] H. Shokrollahi and K. Janghorban: "Soft magnetic composite materials (SMCs),” J. Mater. Process. Technol. 189 (2007) 1 (DOI: 10.1016/j.jmatprotec. 2007.02.034).

[7] I. G. Lee, et al:: "Design of a patterned soft magnetic structure to reduce magnetic flux leakage of magnetic induction wireless power transfer systems," IEEE Trans. Electromagn. Compat. 59 (2017) 1856 (DOI: 10.1109/TEMC. 2017.2690967).

[8] N. Engheta and R. W. Ziolkowski: Metamaterials: Physics and Engineering Explorations (Wiley, New York, 2006).

[9] J. B. Pendry, et al.: "Magnetism from conductors and enhanced nonlinear phenomena," IEEE Trans. Microw. Theory Techn. 47 (1999) 2075 (DOI: 10. 1109/22.798002).

[10] Y. Zhao and E. Leelarasmee: "Controlling the resonances of indefinite materials for maximizing efficiency in wireless power transfer," Microw. Opt. Technol. Lett. 56 (2014) 867 (DOI: 10.1002/mop.28212).

[11] G. Lipworth, et al:: "Magnetic metamaterial superlens for increased range wireless power transfer," Sci. Rep. 4 (2014) 3642 (DOI: 10.1038/srep03642).

[12] A. Rajagopalan, et al:: "Improving power transfer efficiency of a short-range telemetry system using compact metamaterials," IEEE Trans. Microw. Theory Techn. 62 (2014) 947 (DOI: 10.1109/TMTT.2014.2304927).

[13] M. J. Chabalko, et al.: "Magnetic field enhancement in wireless power with metamaterials and magnetic resonant couplers," IEEE Antennas Wireless Propag. Lett. 15 (2016) 452 (DOI: 10.1109/LAWP.2015.2452216).

[14] Y. Cho, et al.: "Thin hybrid metamaterial slab with negative and zero permeability for high efficiency and low electromagnetic field in wireless power transfer systems," IEEE Trans. Electromagn. Compat. 60 (2018) 1001 (DOI: 10.1109/TEMC.2017.2751595).

[15] M. Boyvat and C. V. Hafner: "Molding the flow of magnetic field with metamaterials: Magnetic field shielding," PIER 126 (2012) 303 (DOI: 10.2528/ PIER12022010).

[16] G. Lipworth, et al.: "Quasi-static magnetic field shielding using longitudinal mu-near-zero metamaterials," Sci. Rep. 5 (2015) 12764 (DOI: 10.1038/ srep12764).

[17] J. Besnoff, et al:: "A frequency-selective zero-permeability metamaterial shield for reduction of near-field electromagnetic energy," IEEE Antennas Wireless Propag. Lett. 15 (2016) 654 (DOI: 10.1109/LAWP.2015.2466172).

[18] H. E. Brian: "Printed inductors and capacitors," Tele-Tech Electronic Industries 14 (1954) 68.

[19] S. A. Cummer, et al: "Q-based design equations and loss limits for resonant metamaterials and experimental validation," IEEE Trans. Antennas Propag. 56 (2008) 127 (DOI: 10.1109/TAP.2007.912959).

[20] R. W. Ziolkowski: "Design, fabrication, and testing of double negative metamaterials," IEEE Trans. Antennas Propag. 51 (2003) 1516 (DOI: 10.1109/ TAP.2003.813622).

[21] A. L. A. K. Ranaweera, et al.: "Experimental investigation of compact metamaterial for high efficiency mid-range wireless power transfer applications," J. Appl. Phys. 116 (2014) 043914 (DOI: 10.1063/1.4891715). 


\section{Introduction}

Wireless charging technology based on wireless power transfer (WPT), which delivers power wirelessly to loads, has recently had wider applications, which include low-power portable devices like mobile phones or wearable products consuming $15 \mathrm{~W}$ or less, AGVs (Automated Guided Vehicles), electric cars, and buses. As the inductive power transfer (IPT) technique shows higher efficiency than other WPT methods, it has been the primary interest in the research and commercialization of low power transfer up to only a few watts. For this reason, the electromagnetic wave has not been considered seriously. However, the applications for long distance and high power transfer are being actively studied, and thus the demand for risk management technology is on the rise, with respect to the influence of magnetic flux leakage on electromagnetic interference and the human body $[1,2,3,4]$.

Existing WPT $(20 \mathrm{kHz}-205 \mathrm{kHz})$ reduce magnetic flux leakage by using ferrite materials, which are magnetic materials that attract magnetic fields into them, to absorb the fields or change their path, thereby inducing high magnetic coupling and enhancing the power transfer efficiency. The ferrite materials have relatively high permeability and resistivity, which results in low power loss. However, they have high weight, volume, and brittleness, and are also difficult to process and apply $[3,4]$. Furthermore, their material properties show more loss at high frequencies. Accordingly, the ferrite materials are applicable only to the low frequency band.

In this situation, a soft magnetic alloy becomes prominent as an alternative shielding material because it has not only high permeability and thermal conductivity but also shows a very low magnetic loss both in low- and high-power transfer systems [5]. To apply the soft magnetic metal, that is, the iron-silicon-nickel alloy to wireless power transfer systems as shielding material, soft magnetic composites (SMCs) need to be fabricated. However, SMCs are made by processing metal flake or powder to reduce the eddy current loss due to high conductivity and adding soft resin to increase volume resistivity. This procedure is very complicated and thus the material is very expensive [6]. Recently, there have been researches on enhancing the disadvantages of the prior art by minimizing the eddy current loss through structural modification of the soft magnetic metal, but there is a limitation in that the structure is optimized according to the type of the coil applied [7].

To mitigate the above material disadvantage of the conventional method of reducing magnetic flux leakage, a new attempt is being made to utilize artificial metamaterials that are fabricated based on a resonance structure arranged repeatedly in a certain period [8]. In connection with wireless power transfer systems, such metamaterials reduce the impact of leaking magnetic fields by modifying the directions of the magnetic fields through any electromagnetic structure where effective permeability can be adjusted for a particular frequency $[8,9,10,11$, $12,13]$, improve the efficiency of power transfer, and have lightweight and thin thickness. These advantages are attracting active research [10, 11, 12, 13, 14].

This study analyzed the shielding performance against the magnetic field according to the permeability of the metamaterial and proposed a design of the metamaterial that could reduce the magnetic flux leakage of wireless power transfer 
systems. The proposed structure realized the Mu-Near-Zero property in a particular frequency band through magnetic resonance that was induced by configuring spiral coils and chip capacitors in parallel on a printed circuit board (PCB). In order to verify the performance of the designed metamaterial, transmission and receiver coils were designed, the proposed structure was applied to the rear of the coils, and then the power transfer efficiency and the reduction of magnetic flux leakage were monitored.

\section{Mu-Near-Zero metamaterial effects on the WPT system}

Metamaterials are electromagnetic structures that can refract and reflect magnetic fields by using artificially designed material properties like negative permittivity (epsilon negative), negative permeability (mu negative) and double negative. In the field of wireless power transfer, research interests are mainly concentrated on improving power transfer efficiency by modifying the effective permeability to use negative permeability $\left(\mu_{r}<0\right)$, which attracts magnetic fields from transmission coils to the center, between the transmission and receiver coils [14].

The refractive index of such metamaterials is determined by the permeability realized in an arbitrary periodical structure. The refraction and reflection of an incident magnetic field can be controlled based on the relationship of refractive indexes between different mediums. Ziolkowski and Heyman [8] thoroughly analyzed this concept mathematically and have shown that in Mu-negative media the refractive index can be negative. Based on the theory of negative refractive phenomena, the relationship between transmittance, reflection and refractive index of an electromagnetic wave can be explained by Fresnel's equations.

As shown in Fig. 1, the reflection and transmission coefficients of an oblique incident magnetic field in a medium can be described as follows.

$$
\begin{gathered}
R_{E_{\|}} \approx\left(\frac{H_{o}^{r}}{H_{o}^{i}}\right)^{2}=\left(\frac{n_{2} \cos \theta_{i}-n_{1} \cos \theta_{t}}{n_{2} \cos \theta_{i}+n_{1} \cos \theta_{t}}\right)^{2} \\
T_{E_{\|}} \approx\left(\frac{H_{o}^{t}}{H_{o}^{i}}\right)^{2}=\left(\frac{4 n_{1} n_{2} \cos \theta_{i} \cos \theta_{t}}{n_{1} \cos \theta_{t}+n_{2} \cos \theta_{i}}\right)^{2}
\end{gathered}
$$

Based on Snell's law, these can be rearranged from the perspective of the incident magnetic field as shown in (3) and (4).

$$
\begin{gathered}
R_{E_{\|}} \approx \frac{n_{2}^{2} \cos \theta_{i}-n_{1} \sqrt{n_{2}^{2}-n_{1}^{2} \sin ^{2} \theta_{i}}}{n_{2}^{2} \cos \theta_{i}+n_{1} \sqrt{n_{2}^{2}-n_{1}^{2} \sin ^{2} \theta_{i}}} \\
T_{E_{\|}} \approx \frac{4 n_{1} \cos \theta_{i} \sqrt{n_{2}^{2}-n_{1}^{2} \sin ^{2} \theta_{i}}}{\left[n_{2}^{2} \cos \theta_{i}+\left(n_{1} / n_{2}\right) \sqrt{n_{2}^{2}-n_{1}^{2} \sin ^{2} \theta_{i}}\right]^{2}}
\end{gathered}
$$

Thus, refractive characteristics of metamaterials can be utilized to control the paths of magnetic fields, which can be incident at diverse angles, and to maximize the reduction of magnetic flux leakage by inducing refraction or reflection in a certain direction $[10,11,12,13,14,15,16]$. The optimal refractive index for reducing the magnetic flux leakage was obtained by analyzing refraction and reflection characteristics according to permeability in (3) and (4). Fig. 2 presents 


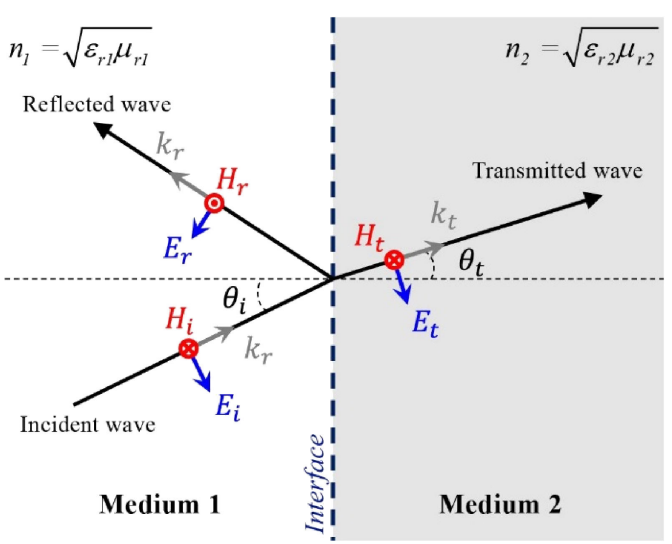

(a)
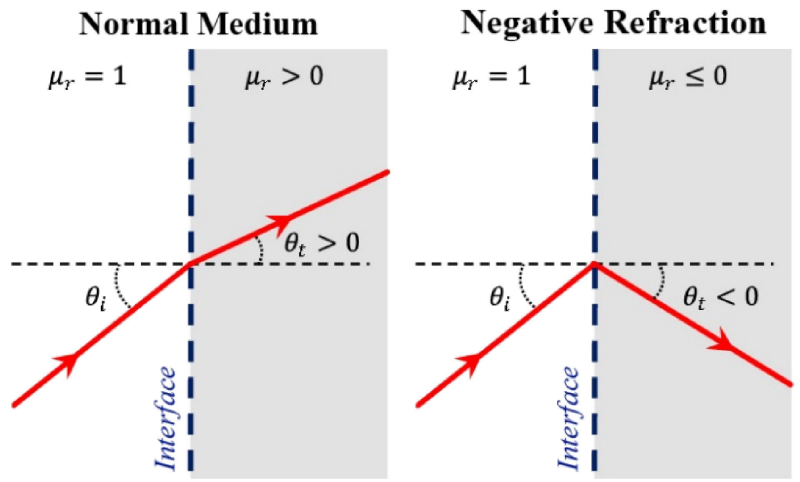

Total Internal Refraction

(b)

Fig. 1. Wave propagation and refractive index (a) Refraction of transverse magnetic wave at the interface between two media (b) Transverse magnetic wave propagation in three different permeability.

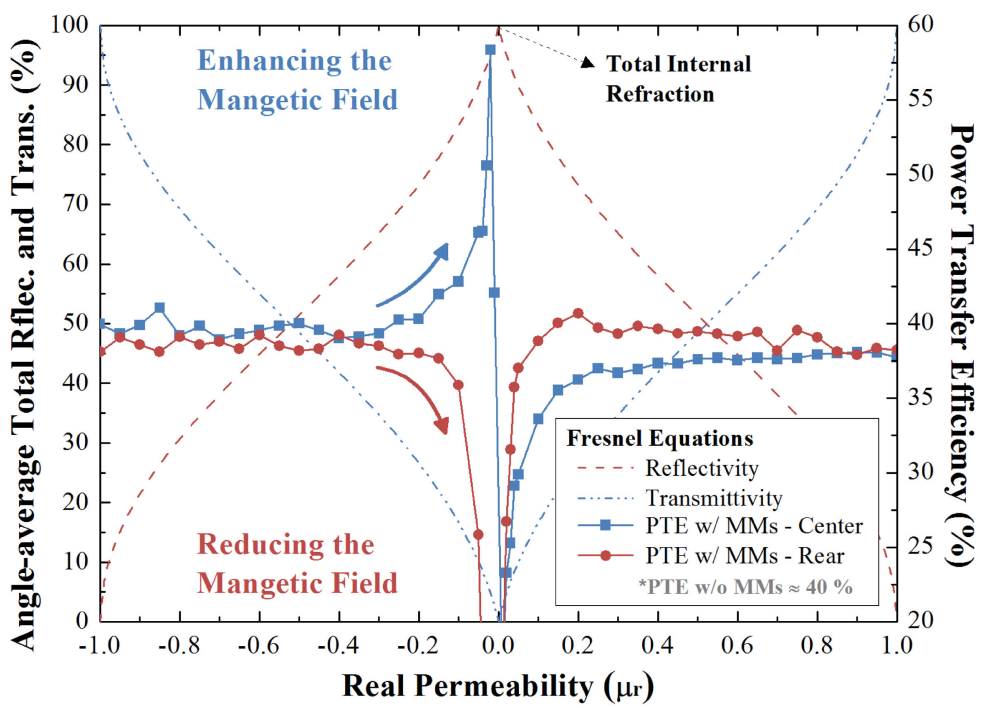

Fig. 2. Calculated angle-average total reflection and transmission, and PTE with metamaterials. 
Before a field simulation and the analysis of frequency characteristics were conducted to check whether the above result was also shown by a wireless power transfer system including the metamaterial, transmission and receiver coils, which were to be used in the simulation, were designed using ANSYS HFSS, a 3D electromagnetic analysis software package. When the coils were fabricated, and the measurement experiment was performed, the frequency characteristics were obtained. These characteristics were compared with the simulation results to verify the performance of the proposed model. Fig. 3(a) shows the power transfer efficiency (PTE) calculated according to different distances between transmission and receiver coils, and Fig. 3(b) presents the measurements (PTE $=\left|S_{21}\right|^{2} \times 100(\%)$ ). To analyze the effect of the metamaterial according to permeability, an isotropic equivalent metamaterial slab (MMs) was assumed at a $0.3 \mathrm{~m}$ distance having maximum magnetic field leakage between transmission and receiver coils [14]. Then, the power transfer efficiency and the distribution of magnetic fields were observed for two cases where the MMs was at the center between the transmission and receiver coils $\left(0.15 \mathrm{~m}\right.$ from the coils, $\left.\mu_{r} \leq 1\right)$ and was at the rear of the coils $(0.03 \mathrm{~m}$ from

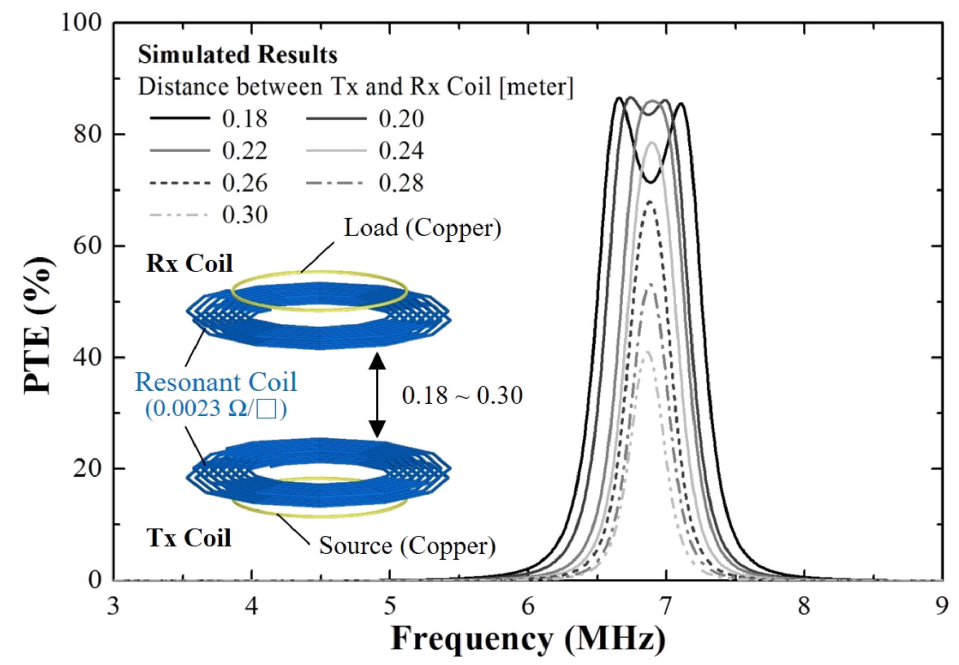

(a)

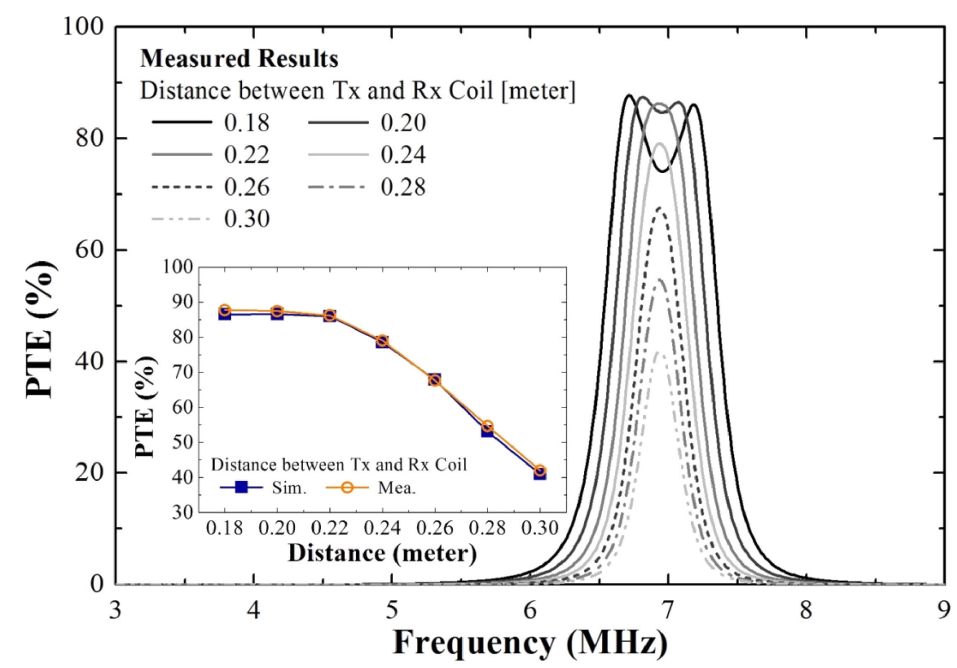

(b) 


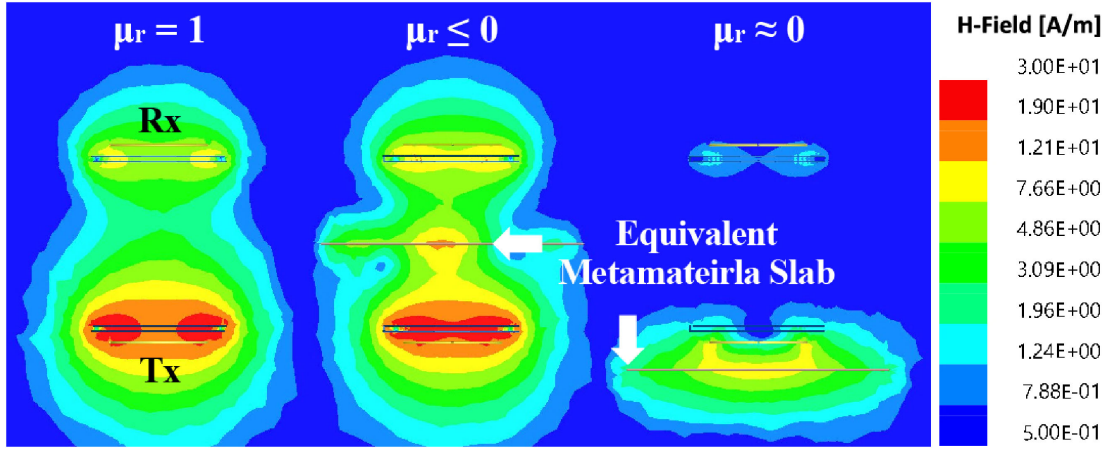

Fig. 4. PTE according to variation of distance between $\mathrm{Tx}$ and $\mathrm{Rx}$ coils.

the coils, $\mu_{r} \approx 1$ ), respectively. Fig. 2 and Fig. 4 illustrate the results in each case. In order to obtain the simulated results of the power transfer efficiency and the distribution of magnetic field, the MMs having the size of $450 \mathrm{~mm} \times 450 \mathrm{~mm}$ with the permeability properties of $\mu_{r}=-1$ and $\mu_{r}=-0.001$ were assumed, respectively.

As shown in Fig. 4, when the MMs was located at the center of the transmission and receiver coils, magnetic fields were focused in the Mu-negative region due to the reverse refraction from the incident direction. Accordingly, the mutual inductance of transmission and receiver coils increased, which results in improvement of the power transfer efficiency $[12,13]$. On the other hand, when the MMs was at the rear of the transmission coil, in the Mu-negative region, as the permeability approached zero (Mu-Near-Zero), the magneto-static volume mode wave (MVW), which interfered magnetic fields generated by the transmission coil, caused a drastic decrease in the power transfer efficiency [17].

Based on the refractive characteristics of the metamaterial, this study applied the metamaterial to the rear of the transmission coil to minimize the degradation of the performance of system. The design parameters of the metamaterial for reducing the magnetic flux leakage were analyzed and the performance of the metamaterial was verified.

\section{Design, simulation and measurement}

In order to design the Mu-Near-Zero property using the magnetic resonance characteristic, the proposed structure arranged square spiral coils in strip lines on a dielectric material $\left(\varepsilon_{r}=4.4, \tan \delta=0.02\right)$ and adopted the CLSR (Capacitively Loaded Square Resonator) structure where chip capacitors were configured in parallel via a hole. With the intent of achieving an optimal design of the metamaterial structure that minimizes Ohmic loss and has a high quality factor of the coil, Bryan's formula (Eq. (5) and (6)) was applied to obtain structural dimensions [18], and inductance (L), resistance (R), and quality factor (Q) were observed according to the variation of coil turns. Here, $D, d, N, w$ and $s$ are the outer diameter, inner diameter, number of turns, line width, and gap distance, respectively. Fig. 5 presents each design parameter, and Table I provides design results. 


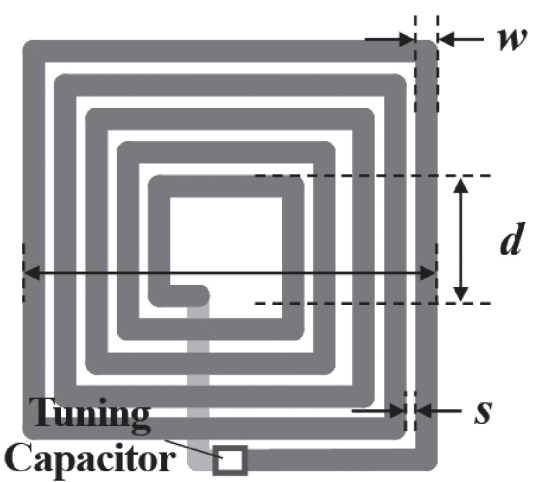

Fig. 5. Design parameters of the metamaterial.

$$
\begin{gathered}
L=6.025 \times 10^{-7}(D+d) \mathrm{N}^{5 / 3} \ln \left[4\left(\frac{D+d}{D-d}\right)\right] \\
D=d+2 N w+2(N-1) s
\end{gathered}
$$

To investigate the electrical characteristics of the metamaterial based on the spiral coil, we performed a simulation observing the characteristics of impedance for calculating the inductance, resistance and quality factor (Q-value). And the capacitance of the capacitor was assumed as a main design parameter for controlling permeability characteristics. Considering imaginary part of relative permeability in Eq. (7), we can confirm that the Q-value affect to the attenuation performance of metamaterial. For these reasons, the nine-turn spiral coil with a high Q-value was adopted to attain the high attenuation characteristics of the metamaterial [16]. Here, $\mu_{r}, \omega_{r}$ and $F$ are the relative permeability, resonant frequency, and geometry dependent factor [19], respectively.

$$
\mu_{r}=1-\frac{F \omega^{2}}{\omega^{2}-\omega_{r}^{2}+j \omega \omega_{r} /(2 Q)}
$$

The permeability was analyzed according to variations of capacitance, the result of which is illustrated in Fig. 6(a). The permeability characteristics of the designed metamaterial were derived from the NRW (Nicolson-Ross-Weir) method using the S-parameter calculated from the Floquet Mode Simulation of HFSS [20].

Table I. Characteristics of WPT coil according to the number of turns

\begin{tabular}{c|c|c|c}
\hline Coil Turns & $\mathrm{L}[\mu \mathrm{H}]$ & $\mathrm{R}[\Omega]$ & $Q$-value \\
\hline 4 & 1.884 & 1.179 & 67.38 \\
\hline 9 & 6.523 & 2.868 & 96.87 \\
\hline 15 & 12.25 & 5.622 & 92.85 \\
\hline
\end{tabular}

Based on the above details of design, a MMs was fabricated which had an $8 \times 8$ arrangement of the unit structure of the nine-turn $(120 \mathrm{pF})$ model having the MuNear-Zero property in the $6.8 \mathrm{MHz}$ band. Frequency characteristics were measured using a test fixture, which consisted of perfect magnetic conductor boundary (ferrite sheet) and perfect electric conductor boundary (copper foil), in order to identify the permeability characteristics of the unit structure of the fabricated MMs. As was done above, the NRW method was used to derive permeability characteristics, 


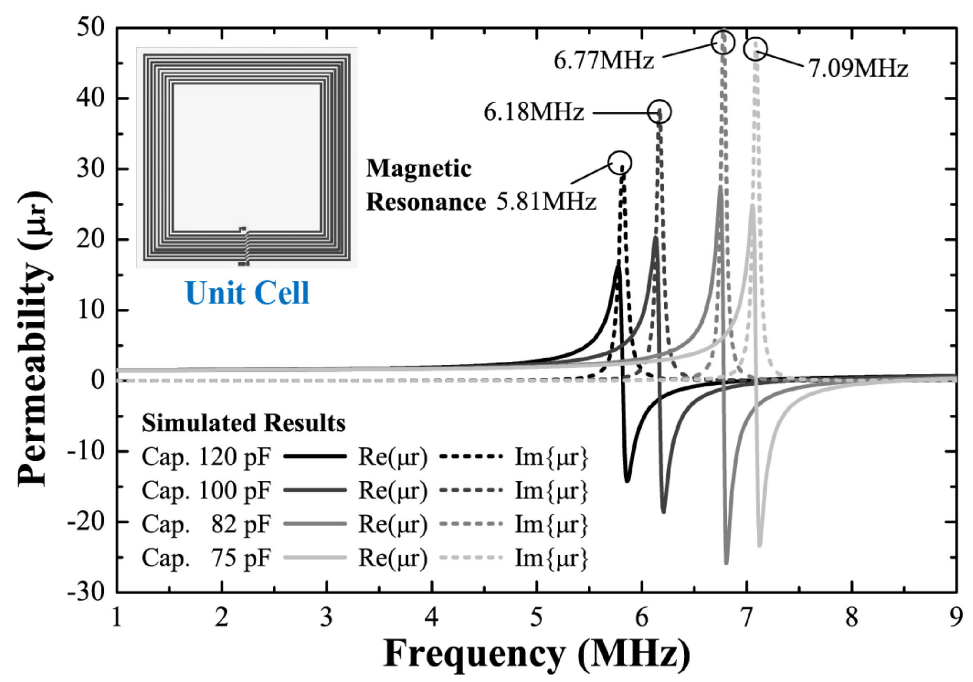

(a)

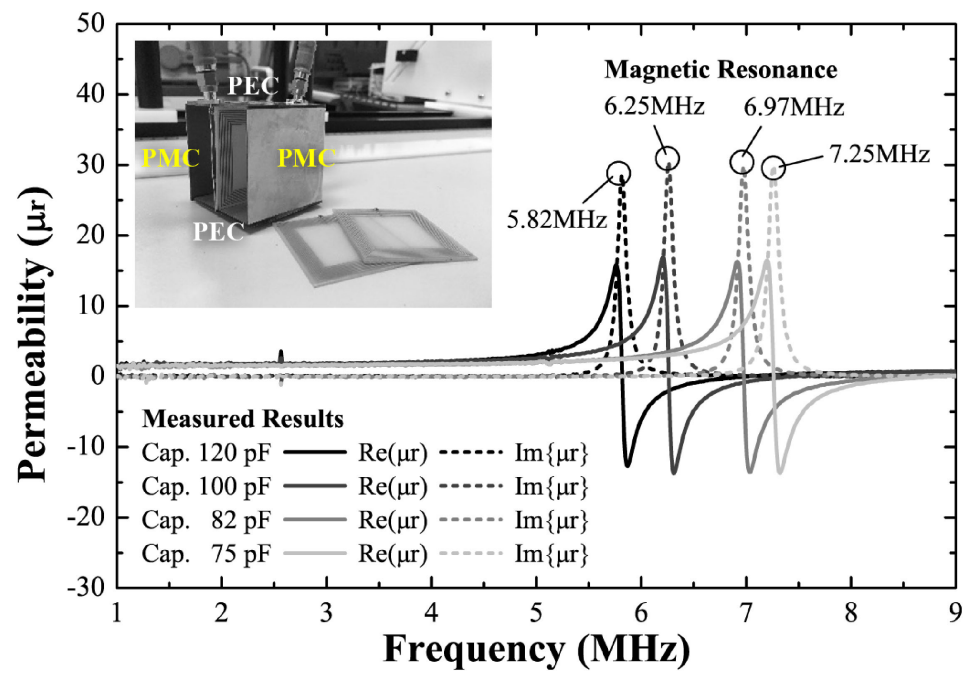

(b)

Fig. 6. Permeability characteristics of proposed metamaterial (a) Simulated results (b) Measured results.

which are shown in Fig. 6(b). To verify the design results, the simulated and measured results were compared by the magnetic resonant frequency of metamaterial. The measurements had good agreement with the simulation results. The errors in the magnetic resonance frequency might be attributable to the difficulty of accurate modeling for the error in the capacitance of real chip capacitors.

Generally, as magnetic resonance-based Mu-negative or Mu-Near-Zero metamaterials satisfy the conditions for MVW caused by the anisotropic material property, these metamaterials tend to decrease the Q-factor of an adjacent coil, thereby degrading the efficiency of the wireless power transfer system [17]. Accordingly, the proposed MMs $\left(\mu_{r}=-0.05\right)$ needs to be located at the rear of the transmission coil in order to reduce the magnetic flux leakage and minimize the degradation of the system efficiency. To determine an optimal distance of the MMs, the power transfer efficiency was analyzed according to different locations of an equivalent slab model with the Mu-Near-Zero property. The analysis results were verified by placing the fabricated $8 \times 8 \mathrm{MMs}$ at the rear of the transmission coil and 
measuring the power transfer efficiency according to the distance of the MMs, while the distance between transmission and receiver coils was $0.3 \mathrm{~m}$. The number of unit structure was established in consideration of the experimental environment and previous studies mentioning the relationship between the number of the unit structure with the performance of MMs [21].

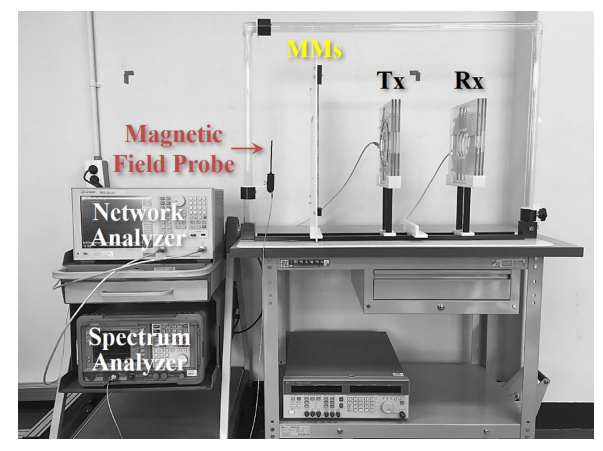

(a)

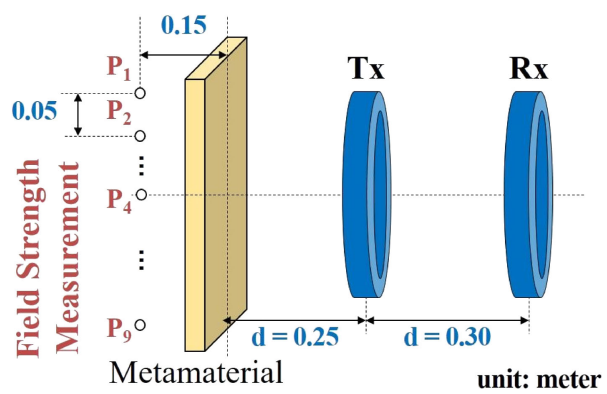

(b)

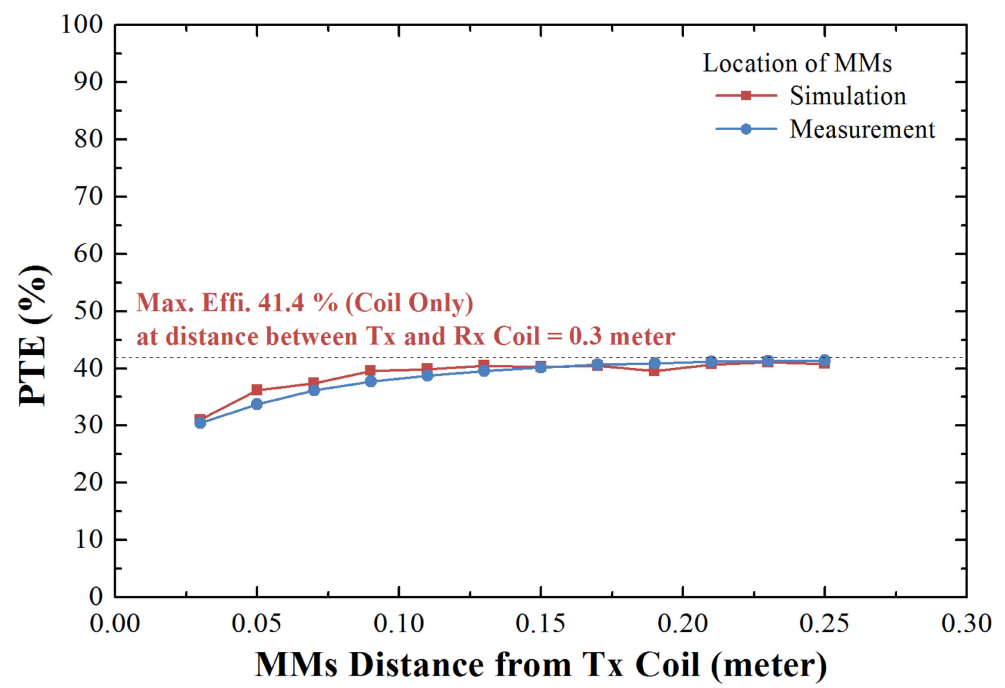

(c)

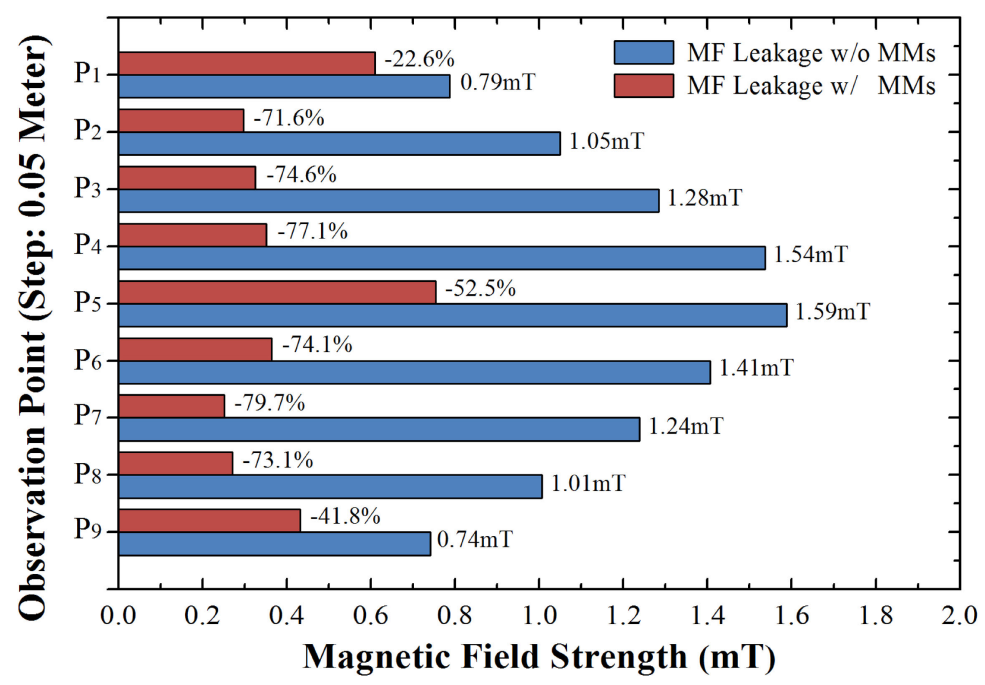

(d) 
Fig. 7(a), (b) illustrates the experimental setup for measuring the power transfer efficiency according to distance, and the magnetic flux leakage at the distance of the metamaterial from the transmission coil where the efficiency degradation was minimized. As shown in Fig. 7(c), when the MMs was farther away from the transmission coil, the power transfer efficiency was less affected. At a distance of $0.25 \mathrm{~m}$ or more, the efficiency $(40.8 \%)$ was close to the case where there was no metamaterial (41.4\%). To verify the performance of reducing magnetic flux leakage, the MMs was placed $0.25 \mathrm{~m}$ from the transmission coil, and the strengths of magnetic fields were measured at each point (step: $0.05 \mathrm{~m}$ ) in the rear of the MMs using the PBS-H2 magnetic flux probe of Aaronia. Fig. 7(d) shows the measurement results. It was found that the magnetic flux leakage could be diminished by a maximum of $79.7 \%$ at the rear of the MMs in a WPT system.

\section{Conclusion}

This study designed a metamaterial that could reduce the magnetic flux leakage at the rear of a transmission coil in a wireless power transfer system. Unlike the existing studies on metamaterials, mostly focusing on efficiency improvement, this study utilized the Mu-Near-Zero permeability property to control the magnetic flux leakage of a wireless power transfer system. Along with the power transfer efficiency of transmission and receiver coils, the strength of the leaking magnetic field was also measured, to minimize the performance degradation of the system due to the metamaterial.

In order to verify the performance of the proposed structure, magnetic resonance transmission and receiver coils, and metamaterials, were fabricated, and the measurement was conducted for the power transfer efficiency and the strength of magnetic flux leakage at the rear of the transmission coil. The results showed that the proposed structure had an efficiency of $40.8 \%$, which was similar to that of the existing wireless power transfer systems and reduced the magnetic flux leakage by a maximum of $79.7 \%$.

The designed metamaterial has not only thin and light but also a good performance for reducing the leakage magnetic field at the certain frequency. However, the metamaterial has some weaknesses such as sufficient distance between transmission coil with metamaterial and size of slab for stable performance is needed. To overcome these limitations, the study on miniaturization of unit structure is needed.

\section{Acknowledgments}

This research was supported by ICT R\&D program through ETRI and by the International Science and Business Belt Program through the Ministry of Science and ICT (2017K000488). 